\title{
Pioneer may collide with Saturn's 'E' ring
}

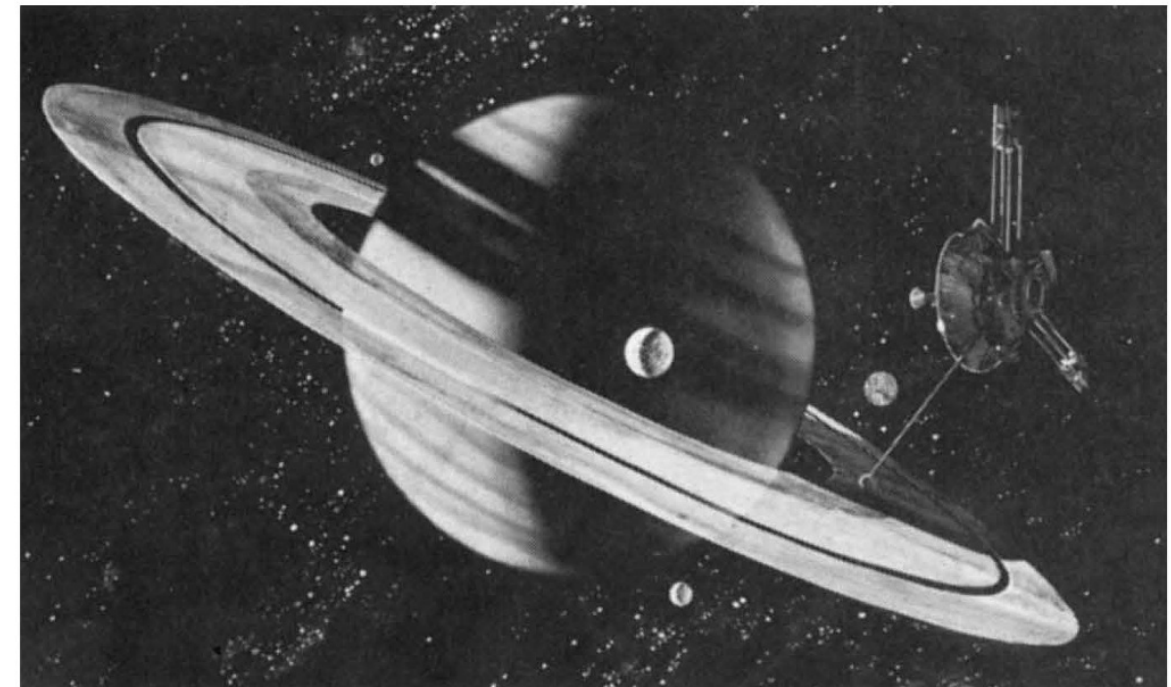

AT 14.35 GMT tomorrow (1 September), the US Pioneer 11 spacecraft will cross the plane of Saturn's rings at an extremely shallow angle, and perhaps find out the hard way whether material in the planet's famous rings is spread out farther than appears on photographs taken from Earth. Because of the time taken for its radio signals to reach Earth, mission scientists waiting anxiously at Ames Research Center in California will not know for more than an hour whether the spacecraft has survived its 0.8 second passage through the ring plane; but the outcome will instantly resolve a long-standing debate over mission strategy - and at least a few private wagers.

There are three clearly visible rings surrounding Saturn: from the outermost inwards they are designated A, B and C. In 1969 , some four years before the launch of Pioneer 11, Pierre Guerin discovered a fourth ring (D) lying even closer to the planet's surface. Mission scientists had originally voted 12 to 1 to have the spacecraft pass through the 'Guerin Division' between the $\mathrm{C}$ and $\mathrm{D}$ rings.

The decision, however, caused a conflict with scientists at the Jet Propulsion Laboratory, whose more sophisticated Voyager spacecraft will be flying by Saturn in November 1980 and August 1981. As its name implies, the Pioneer mission is a relatively low-budget scouting expedition, and JPL scientists wanted to make sure that the subsequent Voyagers would be following a safe path through potentially hostile territory. (Such lapses into the terminology of the Old West are understandable among scientists at Ames, where the laboratory facilities have been built on the site of an old stagecoach stop.)

The dispute was finally settled by officials at NASA headquarters in Washington, who decided to forego the advantages of having Pioneer pass through the Guerin Division only $14,400 \mathrm{~km}$ above the planet's surface. They estimated there would be too much chance of encountering material within the division. Instead, they targeted the spacecraft to cross the ring plane at the presumably safe distance of $112,000 \mathrm{~km}$, well outside the A ring which extends to only $77,400 \mathrm{~km}$. After passing through the ring plane for the first time, Pioneer will approach within $21,400 \mathrm{~km}$ of Saturn's southern hemisphere, then cross the ring plane again at the same height.

Although this plan is still in effect, new data have revealed that even this 'outer option' may not be as safe as originally anticipated. Radar measurements indicate

\section{By John Douglas, California}

that there may be substantial material extending as far as $343,000 \mathrm{~km}$ from Saturn's surface, in a band now being tentatively called the E ring. This material does not appear strongly on ordinary photographs, probably because the E ring is composed of widely scattered large chunks, which can be more easily detected by radar.

When asked whether he thought the Pioneer 11 spacecraft would survive both its passages through the ring plane, chief mission scientist John Wolfe estimated the chances of survival at about $50 \%$. If the spacecraft should hit a sizeable chunk of debris as it passed the rings it could well be destroyed, he said, but more than threequarters of the experiments intended for the mission would already have been completed. In particular, Ames scientists would have photographs of Saturn with a resolution a factor of 10 better than can be obtained with the best Earth-based telescopes.

If Pioneer 11 does not survive its crossing of the ring plane, the greatest loss to the mission will be the proposed encounter with Saturn's principle moon. Titan, the largest known planetary satellite in the solar system, has a thick atmosphere, and there has been much speculation over whether it may be able to support life. Pioneer 11 is not instrumented adequately to fully answer this question, but it has been designed to make some relevant measurements.

In particular, Pioneer instruments should be able to characterise Titan's atmosphere, by determining its opacity, and the composition and layering of its aerosols. Previous Earth-based observations have revealed that Titan's upper atmosphere contains an orangecoloured layer of smog, which could be due to organic compounds. Pioneer's measurements of particle bombardment will indicate whether methane in Titan's atmosphere is being dissociated in a way likely to form organic polymers. The presence of ammonia will also be sought, since nitrogen is a constituent of the reddish organic polymers thought to exist in the smog layer.

Finally, the spacecraft will measure the heat balance of Titan. The surface of the satellite is thought to be too cold (perhaps $-193^{\circ} \mathrm{C}$ ) for life to survive, but it has been suggested that there may be a warm layer in the atmosphere, caused by the greenhouse effect or volcanic action, where the evolution could have occurred.

At the very least, the Pioneer flyby of Saturn should produce some spectacular photographs of considerable scientific interest. Because of the spacecraft's speed, the rings will photograph only as white blurs, although other instruments on board may determine the size of the chunks of rocks and ice which constitute the rings. If Pioneer survives the passage through the ring plane, there should also be a startling visual effect as the rings suddenly reverse in brightness. During its approach, Pioneer will view the rings against the Sun, and they should appear dark, with the gaps bright because of scattering by the small amount of material resident there. After the ring plane passage, the rings will suddenly revert to their normal appearance.

Whatever happens during the crucial encounter with the ring plane, Pioneer will have lived up to its name, for JPL scientists will almost certainly use the findings of this preliminary mission to target the Voyager spacecraft. Meanwhile, Ames scientists have begun to argue that such pathfinder missions will continue to be valuable. Only four months ago, NASA headquarters announced that future planetary missions would be handled entirely by JPL; but since then the top administration of the agency has shifted and the new administration has stated that no laboratory will be automatically excluded from any future missions. It is now reconsidering Ames approach of low-budget exploratory missions as a viable option for future interplanetary exploration, particularly in view of the team's record in successfully controlling costs. The Saturn encounter may well provide the needed impetus for continuing this approach . 